\title{
Retrievable inferior vena cava filter use in trauma: has the fever broken?
}

\author{
Uso de filtro temporário em veia cava inferior em traumas: \\ a febre passou?
}

\section{Raul Coimbra, ${ }^{1}$ Todd Constantini ${ }^{2}$}

Thromboembolic disease in the form of deep venous thrombosis (DVT) and pulmonary embolism (PE) continues to be a major cause of morbidity and mortality following trauma. In the United States, the incidence of nonfatal PE ranges from 450,000 to 650,000 cases per year with an estimation of 50,000 to 200,000 deaths annually. ${ }^{1}$ DVT prophylaxis with unfractionated heparin, low-molecular weight heparin, and sequential compression devices have been used in an attempt to decrease the incidence of DVT, and thus the occurrence of PE. In the absence of major contraindication, the preferred method is anticoagulant thromboprophylaxis. The population of trauma patients at the highest risk for pulmonary embolism is that of patients with lower extremity fractures who are unable to bear weight, with an incidence of DVT as high as $67 \%$ in the absence of prophylaxis. Other patients at increased risk for the development of DVT and PE include those sustaining spine fractures, spinal cord injury, and severe traumatic brain injury.

Due to the nature of their injuries, acute trauma patients frequently will have contraindications to pharmacolo- gical prophylaxis because of the potentially devastating effects of anticoagulant thromboprophylaxis, namely worsening of intracranial hemorrhage and continued posttraumatic internal bleeding. Therefore, the search for additional protection against thromboembolism led to the development of inferior vena cava (IVC) filters. ${ }^{2,3}$

Vena cava filters are indicated to prevent PE in patients with DVT who have contraindication to anticoagulation. Although the indications for IVC filter placement have expanded over the years, DVT or PE in a patient for whom anticoagulation therapy is contraindicated remains the most frequent indication. The most common contraindications to anticoagulation therapy are related to risk of central nervous system hemorrhage and risk of exsanguination. This includes conditions such as severe head injury with intracranial hemorrhage, epidural catheter or hematoma, ongoing hemorrhage or coagulopathy, intraabdominal solid organ injury managed non-operatively, and spinal column fracture. ${ }^{4}$ IVC filter placement is being used increasingly in patients who are at high risk for

\footnotetext{
1. MD, PhD. The Monroe E. Trout Professor of Surgery. Chief, Division of Trauma, Surgical Critical Care, and Burns, Department of Surgery, University of California, San Diego School of Medicine, San Diego, CA, USA.

2. MD. Division of Trauma, Surgical Critical Care, and Burns, Department of Surgery, San Diego School of Medicine, University of California, San Diego, CA, USA.

No conflicts of interest declared concerning the publication of this editorial. 
anticoagulant therapy due to an unsteady gait or tendency to fall, or those patients with poor compliance with medications.

Although not recommended by the American College of Chest Physicians (ACCP) Consensus, ${ }^{5}$ there has recently been a marked increase in the use of retrievable IVC filters in severely injured patients classified as "high risk" for venous thromboembolism. The "prophylactic" indication has contributed to a marked increased, and somehow indiscriminate, use of retrievable IVC filters. Patients with retrievable IVC filters can be considered for filter retrieval when the indication for caval filtration is no longer present or the risk of PE is acceptably low because of a change in clinical status. Removal of the filter can spare the patient from the potential late complications related to long term IVC filter placement, including IVC thrombosis, filter migration, and filter entrapment.

The ability to remove an IVC filter when its use is no longer indicated is clearly ideal. Unfortunately, current clinical experience suggests that the retrieval rate for IVC filters is quite low. The American Association for the Surgery of Trauma (AAST) recently conducted a multi-institutional trial with the purpose of describing practice patterns and outcomes of posttraumatic retrievable filter placement in the United States. ${ }^{6}$ During a 1-year period, 446 patients underwent retrievable IVC filter placement in 21 institutions. Follow-up after discharge was reported in $51 \%$ of patients, but the overall retrieval rate was only $22 \%$. Interestingly, retrieval was 6 -fold higher in patients scheduled for follow-up with the service that placed the retrievable filter. They concluded that most retrievable IVC filters are in fact not retrieved and suggest that the service placing the filter should be responsible for follow-up appointments in order to increase the rate of retrieval.

IVC filter removal may be deferred for various reasons, such as poor clinical status, short patient lifespan, significant amount of clot trapped within the filter, IVC thrombosis, or technical difficulties that prevent safe retrieval. Retrievable IVC filters may be left in place and func- tion as permanent devices, presumably with the same types and rates of complications as permanent devices. ${ }^{7}$ However, these assumptions have not been confirmed by well-designed, long-term studies attempting to define the natural history and long-term complications of these temporary devices.

The majority of acute trauma patients who have received an IVC filter will have their indication for filter placement resolved over time, and should be followed closely to determine if filter removal is appropriate. Patients should be fully evaluated based on clinical status, laboratory findings, and imaging before the attempted retrieval. Clearly, the service placing the device must take the lead in the postdischarge management of retrievable IVC filters. This requires coordinating appropriate follow-up and assuring that filters are removed promptly when clinically indicated. While the "fever" of retrievable IVC filter placement has not broken, surgeons must make the timely removal of the retrievable IVC filter a priority following discharge. In fact, most surgeons should indicate alternatively mechanical prophylaxis with intermittent pneumatic compression or graduate compression stockings, which are the recommendation of the ACCP Consensus.

\section{References}

1. Clagett GP. Basic data related to venous thromboembolism. Ann Vasc Surg. 1988;2:402-5.

2. Cherry RA, Nichols PA, Snavely TM, David MT, Lynch FC. Prophylactic inferior vena cava filters: do they make a difference in trauma patients? J Trauma. 2008;65:544-8.

3. Rogers FB, Shackford SR, Wilson J, Ricci MA, Morris CS. Prophylactic vena cava filter insertion in severely injured trauma patients: indications and preliminary results. J Trauma. 1993;35:637-42.

4. Cuschieri J, Freeman B, O'Keefe G, et al. Inflammation and the host response to injury a large scale collaborative project: patient-oriented research core standard operating procedure for clinical care X. Guidelines for venous thromboembolism prophylaxis in the trauma patient. J Trauma. 2008;65:94450.

5. Geerts, WH, Bergqvist D, Pineo GF, et al. Prevention of venous thromboembolism: American College of Chest Physicians Evidence-Based Clinical Practice Guidelines (8th edition). Chest. 2008;133:381S-453S. 
6. Karmy-Jones R, Jurkovich GJ, Velmahos GC, et al. Practice patterns and outcomes of retrievable vena cava filters in trauma patients: an AAST multicenter study. J Trauma. 2007;62:17-25.

7. Greenfield LJ, Cho KJ, Proctor MC, Sobel M, Shah S, Wingo J. Late results of suprarenal Greenfield vena cava filter placement. Arch Surg. 1992;127:969-73.

\author{
Correspondence: \\ Raul Coimbra, MD, PhD, FACS \\ Division of Trauma, Surgical Critical Care, and Burns \\ Department of Surgery \\ University of California San Diego School of Medicine \\ 200 W. Arbor Drive, $\# 8896$ \\ 92103-8896 - San Diego, CA \\ Tel.: +1 (619) 543.7100 \\ Fax: +1 (619) 543.7202 \\ E-mail: rcoimbra@ucsd.edu
}

